\title{
On-chip constructive cell-network study (II): on-chip quasi-in vivo cardiac toxicity assay for ventricular tachycardia/fibrillation measurement using ring-shaped closed circuit microelectrode with lined-up cardiomyocyte cell network
}

\author{
Fumimasa Nomura, Tomoyuki Kaneko, Akihiro Hattori and Kenji Yasuda*
}

\begin{abstract}
Backgrounds: Conventional in vitro approach using human ether-a-go-go related gene ( $h E R G$ ) assay has been considered worldwide as the first screening assay for cardiac repolarization safety. However, it does not always oredict the potential QT prolongation risk or pro-arrhythmic risk correctly. For adaptable preclinical strategiesto evaluate global cardiac safety, an on-chip quasi-in vivo cardiac toxicity assay for lethal arrhythmia (ventricular tachyarrhythmia) measurement using ring-shaped closed circuit microelectrode chip has been developed.

Results: The ventricular electrocardiogram (ECG)-like field potential data, which includes both the repolarization and the conductance abnormality, was acquired from the self-convolutied extracellular field potentials (FPs) of a lined-up cardiomyocyte network on a circle-shaped microelectrode in an agarose microchamber. When Astemisol applied to the closed-loop cardiomyocyte network, self-convoluted FP profile of normal beating changed into an early afterdepolarization (EAD) like waveform, and then showed ventricular tachyarrhythmias and ventricular fibrilations (VTNf). QT-prolongation-like self-convoluted FP duration prolongation and its fluctuation increase was also observed according to the increase of Astemizole concentration.

Conclusions: The results indicate that the convoluted FPs of the quasi-in vivo cell network assay includes both of the repolarization data and the conductance abnormality of cardiomyocyte networks has the strong potential to prediction lethal arrhythmia.
\end{abstract}

\section{Findings}

Lethal arrhythmia has been one of the major safety concerns for the pharmaceutical industry in selecting and developing compounds. Hence, effects of compounds on the cardiovascular system like blood pressure, heart rate, and electrocardiogram should be assessed appropriately $[1,2]$. Integrated assay systems using human ether-a-gogo related gene (hERG)-transfected HEK-293/CHO-cells (hERG assay), isolated animal tissues (APD or MAPD assay) and conscious and/or anesthetized whole animals (QT or MAPD assay), are currently used to identify QT

\footnotetext{
* Correspondence: yasuda.bmi@tmd.ac.jp Department of Biomedical Information, Division of Biosystems, Institute of Biomaterials and Bioengineering, Tokyo Medical and Dental University, 2-310 Kanda-Surugadai, Chiyoda, Tokyo 101-0062, Japan
}

prolongation [3-5]. Those assay systems are useful to predict QT prolongation risk (inhibition of repolarization process) and conductance's abnormalities. However, they cannot fully predict the potential pro-arrhythmic activities such as Torsades de Pointes (TdP), ventricular tachycardia (VT) or ventricular fibrillation (Vf) induced by compounds [6-8]. In this context, there is a longstanding and urgent need for a surrogate marker that can distinguish the torsadogenic potential from the QT interval duration.

We here propose a quasi-in vivo cardiac toxicity assay, which is a new in-vitro cell network assay technology platform where on-chip technology is used as an assay tool to bridge the gap between conventional in vitro single-cellbased studies and in vivo human clinical settings in terms 
of cardiac toxicity of new chemical entities for drug development. Potential advantages of the proposed strategy of our quasi-in vivo assay to predict lethal arrhythmia (TdP/ $\mathrm{VT} / \mathrm{Vf}$ ) by evaluation of spatial cell-to-cell conductance fluctuation using the on-chip cell network loop which can choose different conductance pathways of human cardiomyocytes among neighboring circulations. We have shown that the on-chip cell network loop model would offer the novel platform to assess the proarrhythmic (not only TdP but also VT/Vf) risks of compounds.

Figure 1 shows the principle and the system set-up of the on-chip quasi-in vivo cell network measurement system. Figure 1(a) shows the relationship of electrophysiological profiles of the single cardiomyocyte extracellular field potential (FP) profile (A), the convoluted FP profiles of lined-up cardiomyocyte network (B), and the surface electrocardiogram (ECG) of individuals (i.e., in vivo surface ECG) (C). The surface ECG is a transthoracic electrical signal of the heart muscle depolarizes during each heart beat externally recorded by skin electrodes. A typical ECG tracing of the cardiac cycle (heartbeat) consists of a P wave, a QRS complex, and a $\mathrm{T}$ wave. ST interval in quasi-ECG model is considered to correspond the duration of $\mathrm{S}$ wave to the apex of $\mathrm{T}$

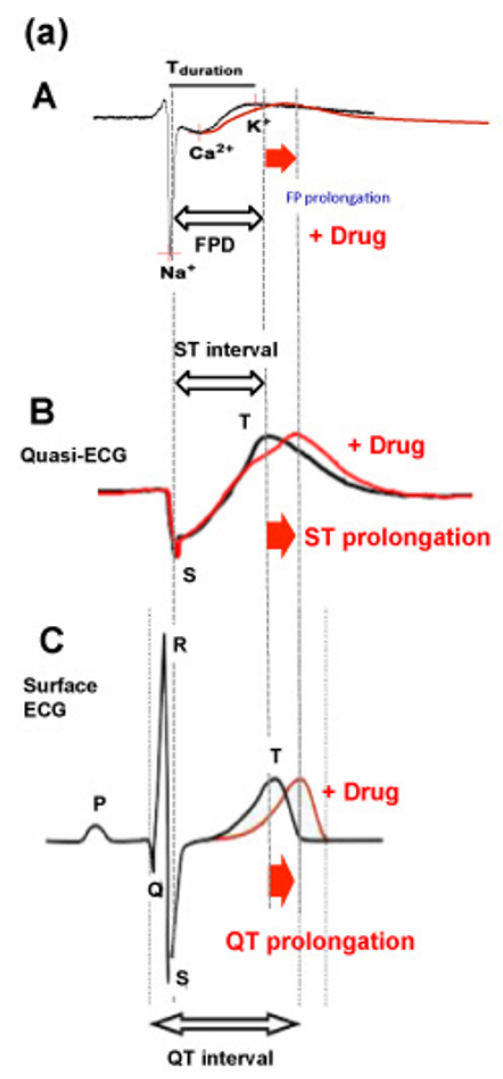

(d)

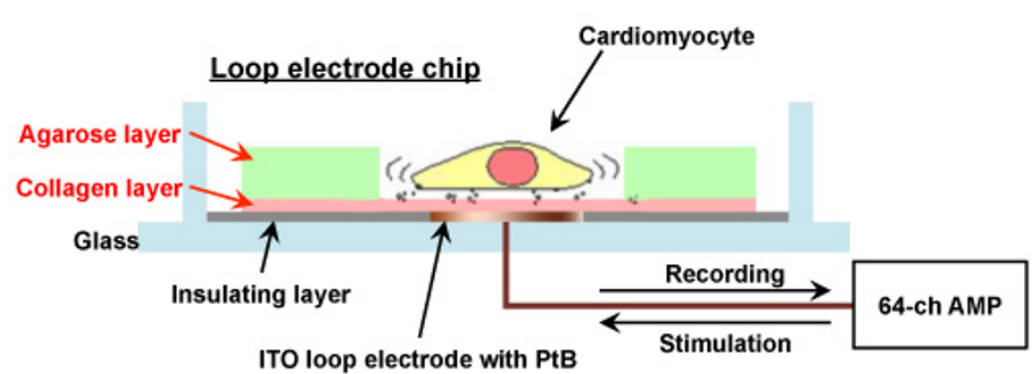

(b)

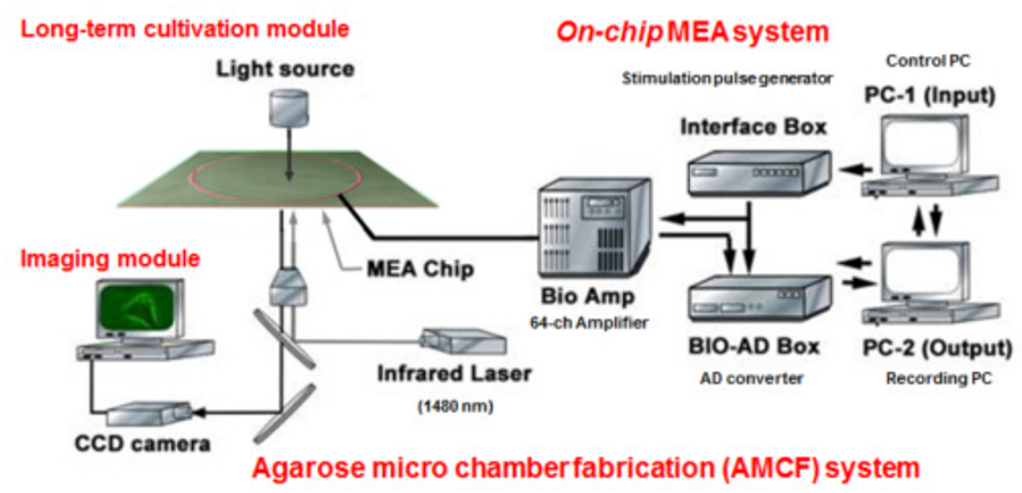

(c)
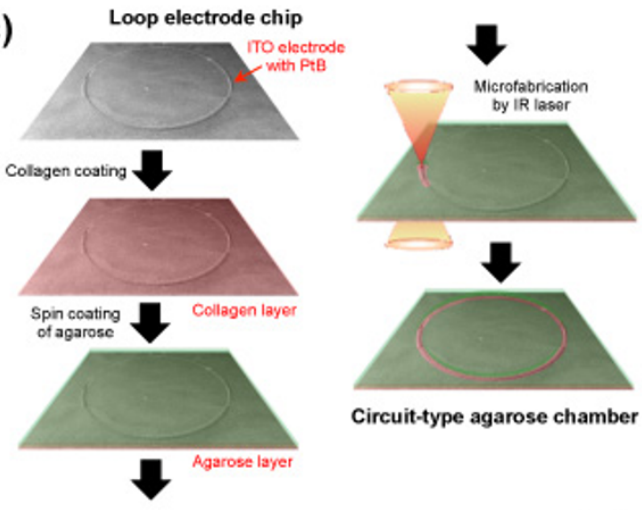

(e)

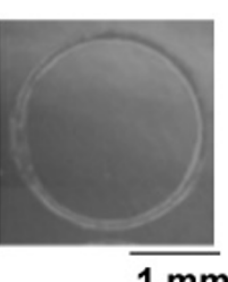

Figure 1 On-chip quasi-in vivo cardiac toxicity measurement assay fabrication. (a) Relationship of field potential profile (FP) of single cardiomyocyte (A), quasi -in vivo ECG signals convoluted from FP profiles of lined-up cardiomyocyte network (B), and the surface electrocardiogram (ECG) of individuals (C). (b) System set-up. (c) Fabrication procedure of closed circuit-shaped lined-up cardiomyocyte network. (d) Crossectional view of loop electrode chip. (e) Phase-contrast image of the ring-shaped closed circuit electrodes. Bar, $1 \mathrm{~mm}$. 


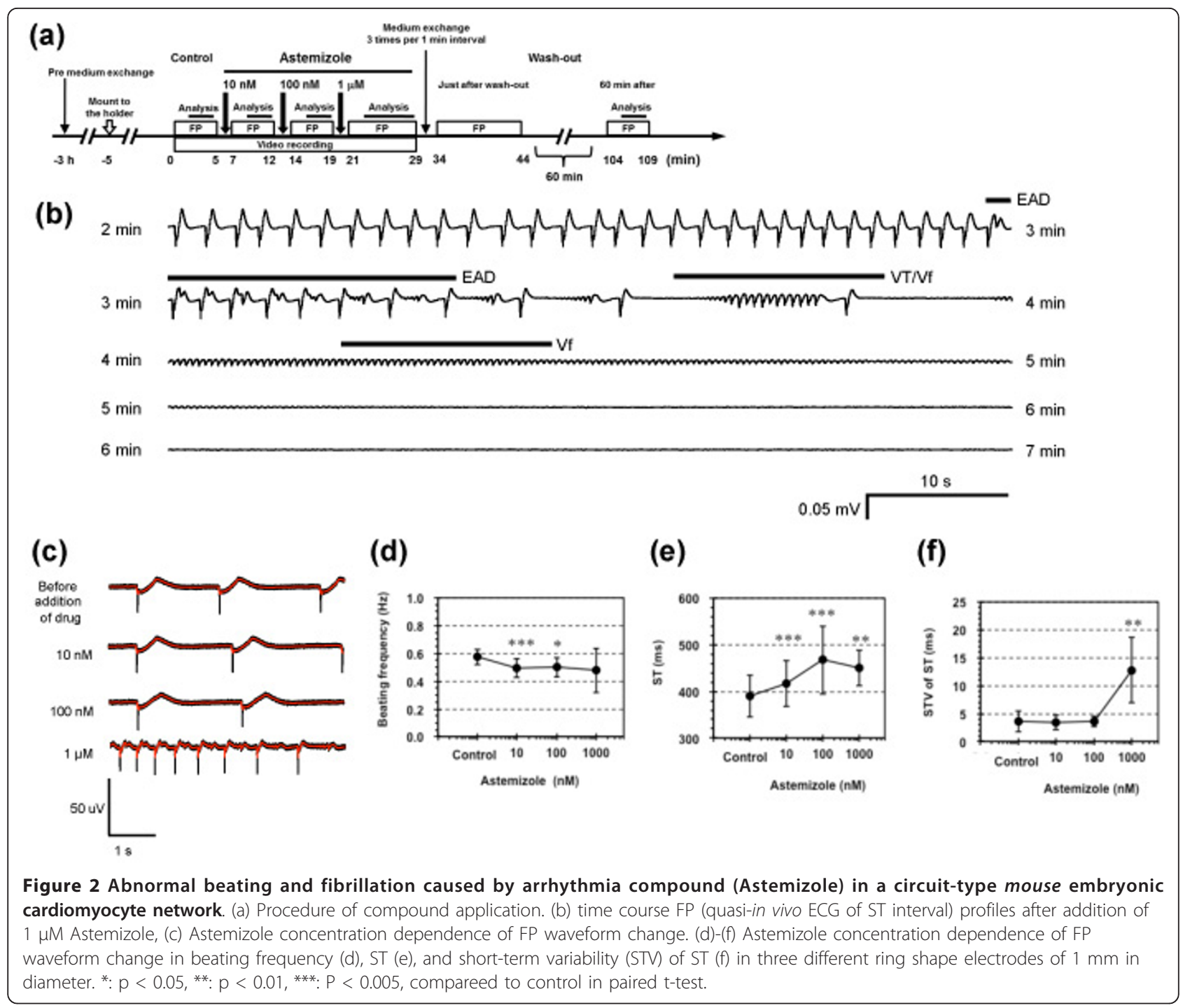

Table 1 Parameter of field potential recordings of cardiomyocytes circuit on ring-type electrodes at the administration of Astemizole

\begin{tabular}{|c|c|c|c|c|c|c|c|c|c|c|c|c|c|}
\hline \multirow[t]{3}{*}{ Sample } & \multicolumn{5}{|c|}{ Beating frequency } & \multicolumn{8}{|c|}{ ST } \\
\hline & \multicolumn{5}{|c|}{ Mean \pm SD $(\mathrm{Hz})$} & \multicolumn{4}{|c|}{ Mean \pm SD (ms) } & \multicolumn{4}{|c|}{ STV (ms) } \\
\hline & Before & $10 \mathrm{nM}$ & $100 \mathrm{nM}$ & $1 \mu \mathrm{M}^{+}$ & & Before & $10 \mathrm{nM}$ & $100 \mathrm{nM}$ & $1 \mu \mathrm{M}^{+}$ & Before & $10 \mathrm{nM}$ & $100 \mathrm{nM}$ & $1 \mu \mathrm{M}^{\dagger}$ \\
\hline 1 & $0.53 \pm 0.04$ & $0.47 \pm 0.06$ & $0.54 \pm 0.06$ & $0.29 \pm 0.09$ & a & $408 \pm 10$ & $427 \pm 9$ & $509 \pm 23$ & $446 \pm 12$ & 7.8 & 6.1 & 4.6 & 9.2 \\
\hline 2 & $0.5 \pm 0.05$ & $0.45 \pm 0.05$ & $0.47 \pm 0.05$ & $0.68 \pm 0.26$ & a & $403 \pm 5$ & $425 \pm 7$ & $481 \pm 12$ & $473 \pm 49$ & 3.4 & 3.1 & 3.3 & 16.9 \\
\hline 3 & $0.63 \pm 0.09$ & $0.54 \pm 0.05$ & $0.52 \pm 0.08$ & $0.47 \pm 0.13$ & a & $364 \pm 6$ & $386 \pm 5$ & $420 \pm 7$ & $441 \pm 27$ & 3.0 & 2.7 & 3.6 & 7.7 \\
\hline 4 & $0.65 \pm 0.06$ & $0.59 \pm 0.05$ & $0.56 \pm 0.05$ & $0.41 \pm 0.09$ & a & $445 \pm 9$ & $491 \pm 14$ & $580 \pm 27$ & $423 \pm 26$ & 2.9 & 3.5 & 4.6 & 21.7 \\
\hline 5 & $0.54 \pm 0.11$ & $0.38 \pm 0.1$ & $0.36 \pm 0.06$ & $0.28 \pm 0.12$ & $\mathrm{~b}$ & $311 \pm 6$ & $337 \pm 4$ & $356 \pm 4$ & $410 \pm 36$ & 1.9 & 1.7 & 2.2 & 5.9 \\
\hline 6 & $0.58 \pm 0.06$ & $0.52 \pm 0.04$ & $0.52 \pm 0.06$ & $0.58 \pm 0.22$ & a & $425 \pm 7$ & $455 \pm 10$ & $497 \pm 7$ & $526 \pm 19$ & 3.6 & 3.6 & 4.0 & 11.0 \\
\hline 7 & $0.53 \pm 0.04$ & $0.47 \pm 0.06$ & $0.54 \pm 0.06$ & $0.29 \pm 0.09$ & a & $371 \pm 8$ & $402 \pm 6$ & $428 \pm 4$ & $435 \pm 54$ & 3.0 & 3.0 & 2.6 & 17.1 \\
\hline Mean & 0.57 & 0.49 & 0.50 & 0.48 & & 390 & 418 & 467 & 451 & 3.7 & 3.4 & 3.5 & 12.8 \\
\hline SD & 0.05 & 0.07 & 0.07 & 0.16 & & 45 & 49 & 73 & 38 & 1.9 & 1.3 & 0.9 & 5.8 \\
\hline t-tset & & $* * *$ & * & & & & $* * *$ & $* * *$ & $* *$ & & & & $* *$ \\
\hline
\end{tabular}

a: Fibrillation after abnormal beating, b: Abnormal beating, t: Calculation from FP recordings just before abnormal beating.

$*^{*}: p<0.05,{ }^{* *}: p<0.01,{ }^{* *}: \mathrm{P}<0.005$, compareed to control in paired t-test. 
wave in surface ECG. Hence the convolution of propagating FP signals in lined-up ventricles cardiomyocyte cell network (B) should represent the characteristics of a piece of ventricles tissue, i.e., quasi-in vivo ECG signals in ventriucles ( $\mathrm{ST}$ interval).

As shown in Figure 1(b), the agarose microchamber fabrication system (AMCF) was used for quasi-in vivo preclinical cardiac toxicity assay, in which extracellular signals (FP) of cardiomyocyte cells in geometrically patterning chambers have been recorded with an multielectrode array (MEA) system [9-13]. For the on-chip quasiin vivo measurement of quasi-in vivo ECG signals, a closed circuit-shaped lined-up cardiomyocyte network was cultivated in a closed circuit-shaped agarose microchamber fitting to the closed loop single electrode, which was fabricated by a spot-heating of a portion of agarose layer as follows (Figure 1(c)): The chip was first coated with collagen Type I-C (Nitta gelatin), and then were spin-coated with $2 \%(\mathrm{w} / \mathrm{v})$ agarose (GenePure LowMelt ISC BioExpress) at $1500 \mathrm{rpm}$ for $20 \mathrm{~s}$. To form the closed circuit chamber for the ring-shaped cell network model, a portion of agarose gel layer on the surface of MEA was removed by the spot heating of focused 1480 $\mathrm{nm}$ infrared laser. Figure 1(d) also shows the cross-sectional schematic drawing of the spatial arrangement of cardiomyocytes in a closed loop-shaped indium tin oxide (ITO) electrode in the quasi-in vivo chip.

Cardiomyocytes were isolated from 13-day-old mouse embryos (ICR) and were placed in agarose micro-chamber on the MEA chip with a concentration having $5 \times 10$ ${ }^{5}$ cells/ml (more than $73.6 \%$ purity of beating cardiomyocytes), and cultivated in a cell culture medium (Invitrogen DMEM supplemented with $10 \%$ fetal bovine serum, $100 \mathrm{U} / \mathrm{ml}$ penicillin, and $100 \mathrm{mg} / \mathrm{mL}$ streptomycin) at $37^{\circ} \mathrm{C}$ with a humidified atmosphere of $95 \%$ air and $5 \% \mathrm{CO}_{2}$. The attached cells on the collagen layer in the agarose micorochamber grew and extended to form the electrophysiologically connected cardiomyocyte within 7 days (Figure 1(e)).

Figure 2 shows the results of quasi-in vivo ECG signals acquired from the closed loop-shaped circuits. To evaluate the prediction ability of ventricular lethal arrhythmia using this cell network assay, Astemizole, which is one of the false-negative compounds on APD prolongation in guinear-pig papillary muscle assay, was applied to the cardiomyocyte networks for VT/Vf measurement with the procedures as shown in Figure 2(a). In Figure 2(b), the time course of FP (quasi-in vivo ECG) waveform changed from a normal beating to an early afterdepolarization (EAD) like waveform, which maintains their beating intervals with abnormal additional depolarization during phase 2 or phase 3 of the cardiac action potential before normal repolarization is competed, and then changed into VT/Vf about $3 \mathrm{~min}$ after $1 \mu \mathrm{M}$ Astemizole application. It should be noted that the EAD and ST interval prolongation started simultaneously in this example. Figure 2(c) shows the example of the FP (quasi-in vivo ECG) waveform of ring-shaped cardiomyocyte network in the short closed loop electrodes ( $1 \mathrm{~mm}$ in diameter) and we confirmed that the length of ST interval apparently prolonged and the fluctuation (short-term variability: STV [14]) of ST duration increased according to the increase of Astemizole concentration.

Figures 2(d) to 2(f), and Table 1 show the summaries of the ring-shaped circuits ( $1 \mathrm{~mm}$ in diameter), indicating beating frequencies (d), ST (e), and STV of ST (f). As shown in the above data, the acquired signals showed ST prolongation and STV increase independent to the circuit diameter differences and similar to the surface ECG signals, but also the occurrence of EAD or subsequent VT/Vf like waveforms, which is similar to the results of in vivo QT screening [15].

There are several advantages of this ring-shaped cell network: First, the round shape is more strong and stable to maintain their spatial cell network arrangements than the linearly lined-up cardiomyocyte network. For example, when we cultivate cardiomyocytes in the linearly lined-up network, the cardiomyocytes at the both ends of linearly lined-up cell network was easily shrunk to the center during their beating because of their force generation and detachment from substrate. Next, as there is no end of cell network in closed loop design, there is no need to arrange pacemaker cardiomyocytes to particular points such as the end of cell network in lined-up model. Third and finally, there is a potential to become a virtual re-entry model, in which the generation of irregular propagation of excitable conduction should be enhanced during its circulation.

The above results also shows the potential of the next generation of on-chip in vitro screening assay using cell network measurements of repolarization and conductance abnormalities for the estimation of delayed repolarization-associated ventricular tachyarrhythmia (e.g., Torsade de Pointes, VT, Vf), which could not be acquired from the in vivo assay such as hERG and APD measurements.

A simple quasi-in vivo ECG measurement assay using closed loop electrode cardiomyocyte network has been developed and the results showed the typical arrhythmia profiles, in which both the temporal repolarization information and the spatial beating propagation information appeared.

\section{Acknowledgements}

This work was financially supported by New Energy Development Organization (NEDO). 


\section{Authors' contributions}

$\mathrm{FN}$, TK and AH carried out whole experiments and participated in the design of the study and contributed to the drafting of the manuscript. KY conceived of the study, participated in its design and coordination and drafted the manuscript. All authors read and approved the final manuscript.

\section{Competing interests}

The authors declare that they have no competing interests.

Received: 9 June 2011 Accepted: 19 September 2011

Published: 19 September 2011

\section{References}

1. $\quad$ CH harmonized tripartite guidelines $S 7 B$ International Conference on Harmonisation of Technical Requirements for Registration of Pharmaceuticals for Human Use; 2005.

2. Bass AS, Darpo B, Breidenbach A, Bruse K, Feldman HS, Garnes D, Hammond T, Haverkamp W, January C, Koerner J, Lawrence C, Leishman D, Roden D, Valentin JP, Vos MA, Zhou Y-Y, Karluss T, Sager P: International Life Sciences Institute (Health and Environmental Sciences Institute, HESI) initiative on moving towards better predictors of drug-induced torsades de pointes. Br J Pharmacol 2008, 154:1491-1501.

3. Hurst JW: Naming of the waves in the ECG, with a brief account of their genesi. Circulation 1937, 98:1937-1942.

4. Takasuna K, Chiba K, Manabe S: Pre-clinical QT risk assessment in pharmaceutical companies - issues of current QT risk assessment Biomol Ther 2009, 17:1-11.

5. Yap YG, Camm AJ: Drug induced QT prolongation and torsades de pointes. Heart 2003, 89:1363-1372.

6. Valentin JP, Pollard C, Lainée P, Hammond T: Value of non- clinical cardiac repolarization. Br J Pharmacol 2010, 159:25-33.

7. Guth BD, Germeyer S, Kolb W, Markert M: Developing a strategy for the nonclinical assessment of proarrhythmic risk of pharmaceutical due to prolonged ventricular repolarization. J Pharmacol Toxicol Methods 2004, 49:159-169.

8. Redfern WS, Carlsson L, Davis AS, Lynch WG, Mackenzie I, Palethorpe S, Siegl PK, Strang I, Sullivan AT, Wallis R, Camm AJ, Hammond TG: Relationships between preclinical cardiac electrophysiology, clinical QT interval prolongation and torsade de pointes for a broad range of drugs: evidence for a provisional safety margin in drug development. Cardiovasc Res 2003, 58:32-45.

9. Moriguchi H, Wakamoto Y, Sugio Y, Takahashi K, Inoue I, Yasuda K: An agar-microchamber cell-cultivation system: flexible change of microchamber shapes during cultivation by photo-thermal etching. Lab Chip 2002, 2:125-130

10. Kojima K, Moriguchi H, Hattori A, Kaneko T, Yasuda K: Two-dimensional network formation of cardiac myocytes in agar microculture chip with 1480-nm infrared laser photo-thermal etching. Lab Chip 2003, 3:299-303.

11. Suzuki I, Sugio Y, Jimbo Y, Yasuda K: Stepwise pattern modification of neuronal network in photo-thermally-etched agarose architecture on multi-electrode array chip for individual-cell-based electrophysiological measurement. Lab Chip 2005, 5:241-247.

12. Kojima K, Kaneko T, Yasuda K: Role of the community effect of cardiomyocyte in the entrainment and reestablishment of stable beating rhythms. Biochem Biophys Res Commun 2006, 351:209-215.

13. Kaneko T, Kojima K, Yasuda K: An on-chip cardiomyocyte cell network assay for stable drug screening regarding community effect of cell network size. Analyst 2007, 132:892-898.

14. Thomsen MB, Verduyn SC, Stengl M, Beekman JDM, de Pater G, van Opstal J, Volders PGA, Vos MA: Increased Short-Term Variability of Repolarization Predicts d-Sotalol-Induced Torsades de Pointes in Dogs. Circulation 2004, 110:2453-2459.

15. Yamamoto K, Tamura T, Imai R, Yamamoto M: Acute canine model for drug-induced Torsades de Pointes in drug safety evaluation-influences of anesthesia and validation with quinidine and astemizole. Toxicol Sci 2001, 60:165-176. doi:10.1186/1477-3155-9-39

Cite this article as: Nomura et al:: On-chip constructive cell-network study (II): on-chip quasi-in vivo cardiac toxicity assay for ventricular tachycardia/fibrillation measurement using ring-shaped closed circuit microelectrode with lined-up cardiomyocyte cell network. Journal of Nanobiotechnology 2011 9:39.

\section{Submit your next manuscript to BioMed Central and take full advantage of:}

- Convenient online submission

- Thorough peer review

- No space constraints or color figure charges

- Immediate publication on acceptance

- Inclusion in PubMed, CAS, Scopus and Google Scholar

- Research which is freely available for redistribution

Submit your manuscript at www.biomedcentral.com/submit
Ciomed Central 